\title{
The Role of a Specific Microbiome in Ovarian Cancer Development
}

\author{
Rafał Karwowski $^{1 *}$, Rafał Kuźlik ${ }^{1}$ and Joanna Gąsiorowska ${ }^{2}$ \\ ${ }^{1}$ Department of Gynecology and Obstetrics, Poland \\ ${ }^{2}$ Departament of Radiology, Poland \\ *Corresponding author: Rafal Karwowski, Department of Gynecology and Obstetrics, Poland
}

ARTICLE INFO

Received: 幽 April 06, 2019

Published: April 15, 2019

Citation: Rafał Karwowski, Rafał Kuźlik, Joanna Gąsiorowska. The Role of a Specific Microbiome in Ovarian Cancer Development. Biomed J Sci \& Tech Res 17(1)-2019. BJSTR. MS.ID.002945.

\begin{abstract}
Microorganisms that exist in the human body can affect the carcinogenesis process even in $20 \%$ of cases. Attempts to determine the relationship between a specific microbiome living in a given environment and the process of cancer formation led to the emergence of a new term-oncobiome. The aim of this study is to present the current state of knowledge about the specific microbiome and its impact on ovarian cancer develepment.
\end{abstract}

Keywords: Microbiome; Oncobiome; Ovarian Cancer

\section{Introduction}

The term microbiome was first used at the turn of the $20^{\text {th }}$ and $21^{\text {st }}$ centuries by the Noble prize winner Joshua Ladeberg and should be understood not only as a description of a simple set of organisms occurring in a given environment, but above all as a set of their genomes interacting with each other and the place where are located [1]. An attempt to detect the dependence between the presence and functioning of a particular microbiome inhabiting the human body on the oncogenesis process is currently one of the main research directions combining such sciences as microbiology, genetics and clinical oncology. Research into the definition of this relationship has in recent years led to the emergence of the term oncobiome, pointing to the direct role and relationship of specific organisms inhabiting the human body to the process of carcinogenesis [2]. It is believed that microorganisms can modulate or influence the formation of up to $20 \%$ of tumors [3]. The example of Helicobacter pylori bacteria, whose eradication from the stomach mucosa allows to significantly reduce the risk of cancer development or vaccination against oncogenic HPV viruses in the prevention of cervical cancer, shows how prospective and future research can assess individual oncobiome living in the human body [4]. Physiologically, the upper part of the female genital organs is a microbiologically sterile environment. Ovaries and the surrounding fallopian tubes may, however, be the site of numerous infections that take place after both acute and chronic infections. The infection may occur, among others through direct spread in the case of infections of nearby organs, eg during appendicitis or salpingitis.
Most infections, however, are ascending and are associated with primary infection of the lower genital tract. Oncobiome research is mainly about colorectal cancer, although in recent years there has been scientific work combining specific microbes and specific microbiota with gynecological tumors. In this article, we conducted a short review of the literature on the microbiome and its connection with ovarian tumors [5-7].

\section{Ovarian Cancer and Pelvic Inflammatory Disease}

Numerous infectious agents that cause pelvic inflammatory disease, both acute and chronic, make the sterile environment of the upper part of the female reproductive system a place exposed to specific, pathogenic flora, both bacterial and viral. For many years, there have been reports of an increased risk of ovarian cancer in the population of women who underwent pelvic inflammatory disease [8,9]. A meta-analysis summarizing 13 case-control studies showed an increased risk of borderline malignancy in women who underwent pelvic inflammatory disease (PID). This risk increased with the increase in the number of PID episodes and was twice as large if the patient underwent PID, at least twice during life [10]. A similar relationship regarding the association of PID episodes with an increased risk of ovarian cancer was noted in a meta-analysis of 6 cohort studies and 7 case-control studies. Serum ovarian cancer occurred statistically significantly more frequently in patients who had an episode of PID in their lifetime [11]. These studies assessed only the general and broadly understood pelvic inflammatory 
disease on the process of oncogenesis in ovarian cancer, and thus the impact of acute and chronic infection of the upper reproductive tract that can be caused by numerous microorganisms. They did not assess the presence of a specific flora, but only indicated the process of chronic inflammation that would initiate the process of tumor formation.

\section{Ovarian Cancer and Viruses}

Most studies on a specific microbiome and its effect on oncogenesis in ovarian cancer relate to human papillomavirusHPV virus. In a meta-analysis conducted in 2013 involving 24 studies on the presence of genetic material of different subtypes of the HPV family virus, a high frequency of viral DNA was found in tumor tissue, thus drawing conclusions about the possible impact of HPV infection on the tumor initiation process [12]. In the studies evaluating genetic material of the virus, the presence of subtypes with high oncogenic potential was found - HPV-16, HPV-18, HPV$45[13,14]$. However, the role of HPV infection in ovarian cancer oncogenesis remains unclear and arouses much controversy among researchers mainly due to data based on few patient groups and data from Asian populations, where the prevalence of HPV infection is definitely higher than in the European population [12]. In a pioneering ovarian cancer oncobiome study published in 2017 researches identified the genetic material of a unique microbiome coexisting with malignant serous tumors using microarrays. In the case of viruses in the analyzed material, the highest hybridization signal was obtained for the Retroviridae Virus signatures, followed by Hepadnaviridae, Papillomaviridae and Flaviviridae. Data published in the cited publication indicate a significant disturbance of the virome present in cancer tissues. Firstly, they indicate completely different families of viruses in the analyzed cancer samples in relation to control samples. Numerous viral signatures, among others Anelloviridae, Astroviridae, Birnaviridae, Bornaviridae, Caliciviridae, Hepadnaviridae were detected at a significant level only in cancer samples. Similarly, in the case of HPV virus, specific molecular signatures of subtypes with high oncogenic potential, i.e. HPV-16 and HPV-18 were detected only in tumor samples and were not found in control group samples [15].

\section{Ovarian Cancer and Bacteria}

The main pathogenic factors associated with ovarian cancer and their potential influence on the oncogenesis process are bacteria-Chlamydia Trachomatis and Mycoplasma Genitalium [16]. They are sexually transmitted bacteria that can cause persistent infections in the upper reproductive tract. Both in the case of $\mathrm{Ch}$. trachomatis and M. genitalium, the suggested mechanism for promoting carcinogenesis is the inhibition of apoptosis in infected cells. Ch. trachomatis can also cause changes in the genome of host cells through the production of reactive oxygen species and the inhibition of dsDNA repair mechanisms $[17,18]$. The presence of Chlamydia Trachomatis genetic material in tissues of invasive ovarian cancer may suggest the effect of infection on the oncogenesis process. In a case-control study conducted in India, the presence of $\mathrm{Ch}$. trachomati DNA was confirmed in $85 \%$ of invasive ovarian cancer samples and the presence of genetic material of this bacterium in control samples including healthy tissue of the female gonad [19]. Similar results were observed in a study published in 2018 [20].

However, the research results are ambiguous. In one of the studies, no genetic material of this microorganism was found in any of the invasive ovarian cancer samples [21]. Similar ambiguous conclusions concern the search for Mycoplasma genitalium DNA. In a study of 27 ovarian cancer samples, this pathogen was demonstrated in 16 of them, which is approx. 59\% [22]. In two other studies, however, no correlation was found between M. genitalium infection and this tumor $[21,23]$. New light on bacterial oncobiome associated with ovarian cancer is being shed by the study already published in 2017 by a group of scientists led by Dr. Sagarika Banerjee. As in the case of viruses, also in the case of bacteria, the results of the research indicated clearly different molecular signatures in tumor tissues as compared to control tissues. The presented study allowed to determine the unique bacterial microbiome present only in cancer samples. The dominant types of bacteria were: Proteobacteria (52\%) and Firmicutes (22\%). Significantly more bacterial signatures were found in tumor tissues as compared to control samples [15].

\section{Ovarian Cancer and Fungi}

The only study that managed to identify the specific fungal microbiota that may be associated with ovarian cancer is the study of the Dr Banerjee group already mentioned. The study detected 18 fungal signatures that were present only in cancerous samples. They were, among others signatures corresponding to Pneumocystis, Acremonium, Cladophialophora, Malassezia and Microsporidia Pleistophora. In contrast, the highest signal in hybridization was obtained for the 18S Cladosporium rRNA [15]. Unfortunately, there are no other studies in which the effect and presence of specific fungal flora in the process of carcinogenesis in ovarian cancer will be assessed.

\section{Summary}

Human microbiome are still discovered in the context of physiological processes taking place in the human body. A look at this world that is not fully understood in the context of pathology of diseases and even their initiation may result in the development of numerous new therapeutic points that could be used not only to treat but also to prevent disease. New research looking for specific and specific microorganisms related to the oncogenesis process indicating numerous quantitative and qualitative disturbances of the microbiome in cancer tissues shows how important influence can be exerted by infectious agents on this process. Already today, some researchers point to the possibility of vaccination against HPV not only in the context of prevention of cervical cancer, but also of ovarian cancer [14]. The detection and linking of specific organisms with the initiation of cancer processes or their promotion would be of particular importance in the case of cancers such as ovarian cancer, which has not been screened so far and whose pathogenesis has remained so far enigmatic. 


\section{References}

1. Lederberg J, Mccray A (2001) Ome Sweet 'Omics -A Genealogical Treasury of Words. The Scientist.

2. Thomas RM, Jobin C (2015) The Microbiome and Cancer: Is the 'Oncobiome' Mirage Real? Trends Cancer 1(1): 24-35.

3. Bhatt AP, Redinbo MR (2017) The role of the microbiome in cancer development and therapy. CA Cancer J Clin 67(4): 326-344.

4. Ford AC (2014) Helicobacter pylori eradication therapy to prevent gastric cancer in healthy asymptomatic infected individuals: systematic review and meta-analysis of randomised controlled trials. BMJ 348 : g3174.

5. Ovarian cancer statistics, American Institute for Cancer Research

6. Zachorowalność

7. https://link.springer.com/content/pdf/bbm\%3A978-3-319-670058\%2F1.pdf

8. Risch HA, Howe GR (1995) Pelvic inflammatory disease and the risk of epithelial ovarian cancer. Cancer Epidemiol Biomarkers Prev 4(5): 447451.

9. Rasmussen CB, Faber MT (2013) Pelvic inflammatory disease and risk of invasive ovarian cancer and ovarian borderline tumors. Cancer Causes Control 24(7): 1459-1464.

10. Rasmussen CB, Kjaer SK (2017) Pelvic Inflammatory Disease and the Risk of Ovarian Cancer and Borderline Ovarian Tumors: A Pooled Analysis of 13 Case-Control Studies. Am J Epidemiol 185(1): 8-20.

11. Zhou Z, Zeng F (2017) Pelvic inflammatory disease and the risk of ovarian cancer: a meta-analysis. Cancer Causes Control 28(5): 415-428.

12. Rosa MI, Silva GD (2013) The prevalence of human papillomavirus in ovarian cancer: a systematic review. Int J Gynecol Cancer 23(3): 437 441.

13. Malisic E, Jankovic R (2012) Detection and genotyping of human papillomaviruses and their role in the development of ovarian carcinomas. Arch Gynecol Obstet 286(3): 723-728.

\section{ISSN: 2574-1241}

DOI: 10.26717/BJSTR.2019.17.002945

Rafał Karwowski. Biomed J Sci \& Tech Res

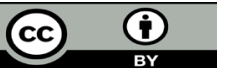

This work is licensed under Creative

Commons Attribution 4.0 License

Submission Link: https://biomedres.us/submit-manuscript.php
14. Al Shabanah OA, Hafez MM (2013) Human papillomavirus genotyping and integration in ovarian cancer Saudi patients. Virol J 10: 343.

15. Banerjee S, Tian T (2017) The ovarian cancer oncobiome. Oncotarget 8(22): 36225-36245

16. Chan PJ, Seraj IM, Kalugdan TH, King A (1996) Prevalence of mycoplasma conserved DNA in malignant ovarian cancer detected using sensitive PCR-ELISA. Gynecol Oncol 63(2): 258-260.

17. Abdul Sater AA, Said Sadier N, Lam VM, Singh B, Pettengill MA, et al. (2010) Enhancement of reactive oxygen species production and chlamydial infection by the mitochondrial Nod-like family member NLRX1. J Biol Chem 285: 41637-4145.

18. Namiki K, Goodison S, Porvasnik S, Robert W Allan, Kenneth A Iczkowski (2009) Persistent exposure to Mycoplasma induces malignant transformation of human prostate cells. Plos One 4: e6872.

19. Shanmughapriya S, Senthilkumar G, Vinodhini K, Das BC, Vasanthi N, et al. (2012) Viral and bacterial aetiologies of epithelial ovarian cancer. Eur J Clin Microbiol Infect Dis. 31(9): 2311-2317.

20. Jonsson S, Oda H (2018) Chlamydia trachomatis, Chlamydial Heat Shock Protein 60 and Anti-Chlamydial Antibodies in Women with Epithelial Ovarian Tumors. Transl Oncol 11(2): 546-551.

21. Idahl A, Lundin E, Elgh F, Jurstrand M, Moller JK (2010) Chlamydia trachomatis, Mycoplasma genitalium, Neisseria gonorrhoeae, human papillomavirus, and polyomavirus are not detectable in human tissue with epithelial ovarian cancer, borderline tumor or benign conditions. Am J Obstet Gynecol 202(1): 7176.

22. Chan P, Seraj I, Kalugdan T, King A (1996) Prevalence of Mycoplasma conserved DNA in malignant ovarian cancer detected using sensitive PCR-ELISA. Gynecol Oncol 63(2): 258-2360.

23. Idahl A, Lundin E, Jurstrand M, Urban Kumlin, Fredrik Elgh, et al. (2011) Chlamydia trachomatis and Mycoplasma genitalium plasma antibodies in relation to epithelial ovarian tumors. Infect Dis Obstet Gynecol 824627.

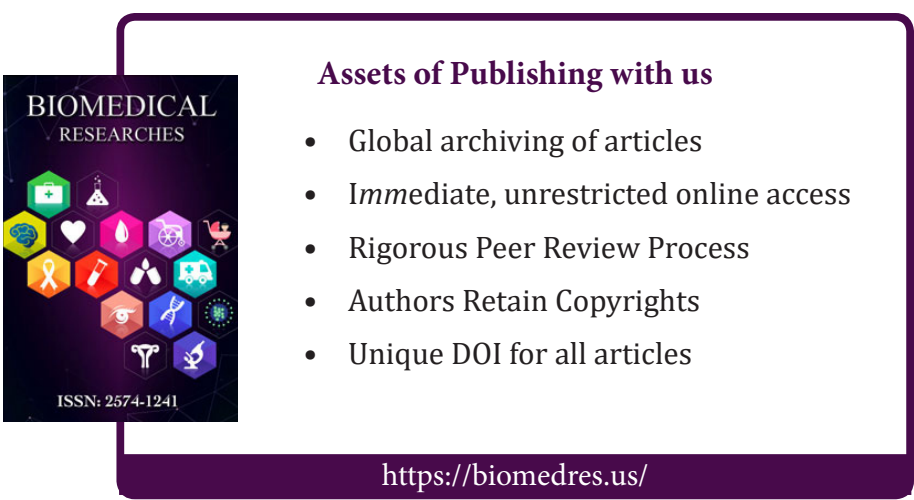

